\title{
Virial theorem in scalar tensor fourth order gravity
}

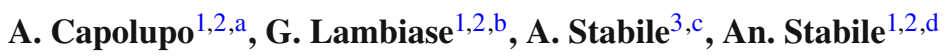 \\ ${ }^{1}$ Dipartimento di Fisica "E.R. Caianiello", Università degli Studi di Salerno, via G. Paolo II, 84084 Fisciano, Italy \\ ${ }^{2}$ Istituto Nazionale di Fisica Nucleare (INFN), Gruppo collegato di Salerno, Salerno, Italy \\ ${ }^{3}$ Dipartimento di Ingegneria, Università del Sannio, Palazzo Dell'Aquila Bosco Lucarelli, Corso Garibaldi, 107, 82100 Benevento, Italy
}

Received: 18 February 2021 / Accepted: 13 July 2021 / Published online: 23 July 2021

(C) The Author(s) 2021

\begin{abstract}
In this paper, we study, in the Newtonian limit, the virial theorem in the context of a scalar tensor fourth order gravity. In particular, we show, that for a isolated galaxy in viral equilibrium, a specific class of scalar tensor fourth order gravity, i.e. $f(R, \phi)+\omega(\phi) \phi_{; \alpha} \phi^{; \alpha}$ in not suitable to explain the large fraction of dark matter necessary to have the flatness of the galaxies rotation curves experimentally observed.
\end{abstract}

\section{Introduction}

Several observational data [1-6] probe that the observed Universe appears in accelerated expansion and spatially flat. To explain these observations it is necessary to introduce two dark components: the dark energy at cosmological scales and the dark matter at galactic and extragalactic scales. In particular, the dynamical evolution of self-gravitating structures can be figured out by the General Relativity, but a not negligible quantity of Dark Matter is needed to obtain agreement with observations [7].

Several authors [8-10] considered some models of extended gravity as a possible theoretical mechanism to explain the galactic rotation curves and cosmic acceleration without introducing any exotic matter. In the context of models of extended gravity, one modifies the gravity sector introducing additional contributions with respect to the standard General Relativity.

In the weak-field approximation, in general, any models of extended gravity yields corrections to the gravitational potential $[11,12]$ which, (using the Solar System as a laboratory) at the Newtonian and post-Newtonian level, could constitute the test-bed for these theories [13-15].

\footnotetext{
a e-mail: capolupo@sa.infn.it

be-mail: lambiase@sa.infn.it

c e-mail: arturo.stabile@gmail.com

de-mail: astabile@unisa.it (corresponding author)
}

From a conceptual viewpoint, there is no reason a priori to restrict the gravitational Lagrangian to a linear function of the Ricci scalar minimally coupled to matter. In particular, one may consider the generalization of $f(R, \phi)$ models, where $R$ is the Ricci scalar and $\phi$ is a massive scalar field Non-minimally to geometry. As models of extended gravity we consider a particular class: scalar tensor fourth order gravity (STFOG). Models of STFOG have been studied widely in the Newtonian and post Newtonian [16-23] limit, as well as in Minkowskian limit [24,25]. Gravitational lensing, stellar hydrodynamics and Galactic rotation curves appear natural candidates as test-bed experiments [26-31]. In this field we have several contributions from various authors [32-44]. An interesting application concerns the study of the Casimir effect [45-47] in weak-field limit in the context of STFOG.

In classic mechanics the virial theorem states that for a stable, self-gravitating, spherical distribution of equal mass objects the total kinetic energy of the objects is equal to minus $1 / 2$ times the total gravitational potential energy. More specifically, the virial theorem provides a general equation that relates in a proportionality of the average over time of the kinetic energy of an isolated gravitational N-body system and bound by a potential force, with the average over time of the potential energy. In general, the virial theorem can be used to connect the average internal motion of the isolated relaxed gravitational system with its dynamic mass.

The aim of this paper is to show that in the Newtonian limit, the virial theorem implies that for an isolated galaxy in viral equilibrium, a specific class of STFOF of the form $f(R, \phi)+\omega(\phi) \phi ; \alpha \phi^{; \alpha}$ is inadequate to explain the large fraction of dark matter which is necessary to account for the flatness of the galaxies rotation curves. Such a result occurs for some values of the parameters characterizing the model under consideration.

The outline of this paper is the following. In Sect. 2 we give the action of a STFOG and write down the corresponding field equations, which we then solve in the presence of 
matter within the weak-field approximation. In Sect. 3 we discuss the extension of the virial theorem in the framework to STFOG. In Sect. 4 we show how the STFOG could simulate dark matter. In Sect. 5 we analyze some models of STFOG. We summarize our conclusions in Sect. 6.

\section{The scalar tensor fourth order gravity}

Let us consider the action:

$$
S=\int d^{4} x \sqrt{-g}\left[f(R, \phi)+\omega(\phi) \phi_{; \alpha} \phi^{; \alpha}+\mathcal{X} \mathcal{L}_{m}\right],
$$

where $f$ is an analytic function of the Ricci scalar $R$ and the scalar field $\phi, \omega$ is a generic function of scalar field while $\mathcal{L}_{m}$ is the minimally coupled ordinary matter Lagrangian density. In the metric approach, namely, when the gravitational field is fully described by only the metric tensor $g_{\mu \nu}$, the field equations are obtained by varying the action with respect the metric, leading to:

$$
\begin{aligned}
& f_{R} R_{\mu \nu}-\frac{f+\omega(\phi) \phi_{; \alpha} \phi^{; \alpha}}{2} g_{\mu \nu}+\omega(\phi) \phi_{; \mu} \phi_{; \nu}-f_{R ; \mu \nu} \\
& \quad+g_{\mu \nu} \square f_{R}=\mathcal{X} T_{\mu \nu}, \\
& 2 \omega(\phi) \square \phi+\omega_{\phi}(\phi) \phi_{; \alpha} \phi^{; \alpha}-f_{\phi}=0, \\
& f_{R} R-2 f-\omega(\phi) \phi_{; \alpha} \phi^{; \alpha}+3 \square f_{R}=\mathcal{X} T .
\end{aligned}
$$

Here $T_{\mu \nu}=\frac{-1}{\sqrt{-g}} \frac{\delta\left(\sqrt{-g} \mathcal{L}_{m}\right)}{\delta g^{\mu \nu}}$ is the energy-momentum tensor of matter, $T=T^{\sigma}{ }_{\sigma}$ is the trace, $R_{\mu \nu}$ is the Ricci tensor, $g_{\mu \nu}$ is the metric tensor, $g=\operatorname{det} g_{\mu \nu}, f_{R}=\frac{\partial f}{\partial R}, f_{\phi}=$ $\frac{\partial f}{\partial \phi}, \omega_{\phi}=\frac{d \omega}{d \phi}, \square={ }_{; \sigma} ; \sigma$ the d'Alembert operator and $\mathcal{X}=8 \pi G$, with $G$ the gravitational constant [16-19]. We assume $c=1$. We are interested in the use of the solutions of the field equations (2) in the case of static (or very slow time depending) and weak gravitational field. In this case the metric tensor $g_{\mu \nu}$ can be thought as:

$g_{00}=1+2 \Phi, \quad g_{i j}=-\delta_{i j}$

and the scalar field as

$\phi=\phi^{(0)}+\varphi$,

where $\Phi, \varphi$ are two scalar functions depending on the spatial variables $\mathbf{x}=\left(x^{1}, x^{2}, x^{3}\right)$ and $\delta_{i j}$ is the Kronecker delta [19]. The energy-momentum tensor $T_{\mu \nu}$ for a perfect fluid, when the pressure is negligible with respect to the mass density $\rho$, is given easily by $T_{\mu \nu}=\rho u_{\mu} u_{\nu}$, with the conditions $u_{\sigma} u^{\sigma}=1$. Finally among all the analytical functions $f$ of Ricci scalar $R$ and scalar field $\phi$, we choose the class of $f$ satisfying the conditions $f\left(0, \phi^{(0)}\right)=0$ and $f_{\phi}\left(0, \phi^{(0)}\right)=0$.
With these hypothesis the field equations (2) become:

$$
\begin{aligned}
& f_{R}\left(0, \phi^{(0)}\right)\left[\triangle \Phi-\frac{R}{2}\right]-f_{R R}\left(0, \phi^{(0)}\right) \triangle R \\
& \quad-f_{R \phi}\left(0, \phi^{(0)}\right) \triangle \varphi=\mathcal{X} \rho, \\
& 2 \omega\left(\phi^{(0)}\right) \triangle \varphi+f_{\phi \phi}\left(0, \phi^{(0)}\right) \varphi+f_{R \phi}\left(0, \phi^{(0)}\right) R=0, \\
& 3 f_{R R}\left(0, \phi^{(0)}\right) \triangle R+f_{R}\left(0, \phi^{(0)}\right) R \\
& \quad+3 f_{R \phi}\left(0, \phi^{(0)}\right) \triangle \varphi=-\mathcal{X} \rho,
\end{aligned}
$$

where $\triangle$ is the Laplacian in the flat space. These equations are not simply the merging of field equations of $f(R)$-gravity and a further massive scalar field, but are due to the fact that the model $f(R, \phi)$ generates a coupled system of equations with respect to Ricci scalar $R$ and scalar field $\phi$ [19]. We note that, for $f_{R R}\left(0, \phi^{(0)}\right)=0, f_{R \phi}\left(0, \phi^{(0)}\right)=0$ and $f_{R}\left(0, \phi^{(0)}\right)=1$, we have the standard Poisson equation: $\triangle \Phi=4 \pi G \rho$. This means that the deviations from GR emerge when the second derivatives of $f$ are different from zero. We introduce the "masses"1 $m_{R}$ and $m_{\phi}$, defined by the conditions:

$m_{R}^{2}=-\frac{f_{R}\left(0, \phi^{(0)}\right)}{3 f_{R R}\left(0, \phi^{(0)}\right)} \quad$ and $\quad m_{\phi}^{2}=-\frac{f_{\phi \phi}\left(0, \phi^{(0)}\right)}{2 \omega\left(\phi^{(0)}\right)}$.

From the field equations (3), we obtain the more general solutions of field equations as follow:

$$
\begin{aligned}
\Phi(\mathbf{x})= & -\frac{\mathcal{X}}{4 \pi f_{R}\left(0, \phi^{(0)}\right)} \int d^{3} \mathbf{x}^{\prime} \frac{\rho\left(\mathbf{x}^{\prime}\right)}{\left|\mathbf{x}-\mathbf{x}^{\prime}\right|} \\
& -\frac{1}{8 \pi} \int d^{3} \mathbf{x}^{\prime} \frac{R\left(\mathbf{x}^{\prime}\right)}{\mid \mathbf{x}} \\
& -\mathbf{x}^{\prime} \mid-\frac{R(\mathbf{x})}{3 m_{R}{ }^{2}}+f_{R, \phi}\left(0, \phi^{(0)}\right) \varphi(\mathbf{x}), \\
\varphi(\mathbf{x})= & -\frac{m_{R}^{2} f_{R \phi}\left(0, \phi^{(0)}\right) \mathcal{X}}{2 \omega\left(\phi^{(0)}\right)} \int \frac{d^{3} \mathbf{k}}{(2 \pi)^{3 / 2}} \\
& \times \frac{\tilde{\rho}(\mathbf{k}) e^{i \mathbf{k} \cdot \mathbf{x}}}{\left(\mathbf{k}^{2}+k_{1}^{2}\right)\left(\mathbf{k}^{2}+k_{2}^{2}\right)}, \\
R(\mathbf{x})= & -\frac{m_{R}{ }^{2} \mathcal{X}}{f_{R}\left(0, \phi^{(0)}\right)} \int \frac{d^{3} \mathbf{k}}{(2 \pi)^{3 / 2}} \frac{\tilde{\rho}(\mathbf{k})\left(\mathbf{k}^{2}+m_{\phi^{2}}\right) e^{i \mathbf{k} \cdot \mathbf{x}}}{\left(\mathbf{k}^{2}+k_{1}^{2}\right)\left(\mathbf{k}^{2}+k_{2}^{2}\right)},
\end{aligned}
$$

where $\tilde{\rho}(\mathbf{k})=\int \frac{d^{3} \mathbf{x}^{\prime}}{(2 \pi)^{3 / 2}} \rho\left(\mathbf{x}^{\prime}\right) e^{-i \mathbf{k} \cdot \mathbf{x}^{\prime}}$, is the Fourier transform of mass density.

Once the mass density is fixed, the gravitational potential $\Phi$ is found by solving the complete set of equations (5). However it is more interesting the direct relation which links the potential with the mass density. The general solution (the

\footnotetext{
${ }^{1}$ In our formalism we consider the coefficients of $R^{2}$ and $\phi^{2}$ negative. For this reason, inside of Eqs. (4) we have the minus signs. However, the definitions of the masses Eqs. (4) are consistent with the formalism used by K. S. Stelle in Ref. [48].
} 
metric tensor components) is written by using the intermediate quantities $\varphi, R$ only for the choice of calculus strategy. Indeed we can rephrase the system (5) eliminating the auxiliary quantities and obtain the generalized Poisson equation in the scalar tensor fourth order gravity non-minimally coupled [22]:

$$
\begin{aligned}
& \Delta \Phi(\mathbf{x})=\frac{16 \pi G \rho(\mathbf{x})}{3 f_{R}\left(0, \phi^{(0)}\right)} \\
& -\frac{m_{R}^{2} G}{f_{R}\left(0, \phi^{(0)}\right)}\left[\mathcal{A}(\xi, \eta) \int d^{3} \mathbf{x}^{\prime} \frac{e^{-m_{+}\left|\mathbf{x}-\mathbf{x}^{\prime}\right|}}{\left|\mathbf{x}-\mathbf{x}^{\prime}\right|} \rho\left(\mathbf{x}^{\prime}\right)\right. \\
& \left.+\left(\frac{1}{3}-\mathcal{A}(\xi, \eta)\right) \int d^{3} \mathbf{x}^{\prime} \frac{e^{-m_{-}\left|\mathbf{x}-\mathbf{x}^{\prime}\right|}}{\left|\mathbf{x}-\mathbf{x}^{\prime}\right|} \rho\left(\mathbf{x}^{\prime}\right)\right]
\end{aligned}
$$

where

$$
\begin{aligned}
\eta & =\frac{m_{\phi}}{m_{R}}, \quad \xi=\frac{3 f_{R \phi}^{2}\left(0, \phi^{(0)}\right)}{2 \omega\left(\phi^{(0)}\right)}, \\
\mathcal{A}(\eta, \xi) & =\frac{1}{6}+\frac{1-\xi-\eta^{2}}{6 \sqrt{\eta^{4}+(\xi-1)^{2}-2 \eta^{2}(\xi+1)}}, \\
\omega_{ \pm}^{2}(\eta, \xi) & =\frac{1-\xi+\eta^{2} \pm \sqrt{\left(1-\xi+\eta^{2}\right)^{2}-4 \eta^{2}}}{2} \\
m_{ \pm}^{2} & =m_{R}^{2} \omega_{ \pm}^{2}(\eta, \xi) .
\end{aligned}
$$

We note in the case of minimally coupled theory $\left(f_{R \phi}=\right.$ $0 \rightarrow \xi=0$ ), we have $m_{+}=m_{R}, m_{-}=m_{\phi}$ and the scalar field $\phi=\phi^{(0)}+\varphi$ is disconnected from the matter source $^{2}$.

In general, the STFOG models present the potential $\Phi$ with massive modes [25]. In fact, in Eq. (6), we find two massive modes, with masses $m_{+}$and $m_{-}$. In the present analysis we assume that these modes have two distinct real masses ${ }^{3}$. For this reason, the parameters $\eta$ and $\xi$ must satisfy the following constraint:

$0 \leqslant \xi \leq(\eta-1)^{2} \quad \forall \eta \geqslant 0$

in order to have real masses (see Fig. 1), i.e. $m_{ \pm}^{2} \geq 0$. Therefore, the constraint (9) on the parameters $\eta$ and $\xi$ restricts the class of theories (1) which can be used.

In the case of a point source, $\rho(\mathbf{x})=M \delta^{(3)}(\mathbf{x}) \delta^{(3)}$, where $\delta^{(3)}$ is the three dimension Dirac delta function, the solution of (6) is [16-19]:

$$
\begin{aligned}
& \Phi_{\text {point }}(\mathbf{x})=-\frac{G M}{f_{R}\left(0, \phi^{(0)}\right)|\mathbf{x}|}\left[1+g(\eta, \xi) e^{-m_{+}|\mathbf{x}|}\right. \\
& \left.+[1 / 3-g(\eta, \xi)] e^{-m_{-}|\mathbf{x}|}\right]
\end{aligned}
$$

\footnotetext{
$\overline{2}$ At newtonian level the dynamical evolution of the scalar field in any non-minimally coupled theory is the same as in any minimally one.

3 From Eq. (7) it is easy to notice that $m_{+}>m_{-}$.
}

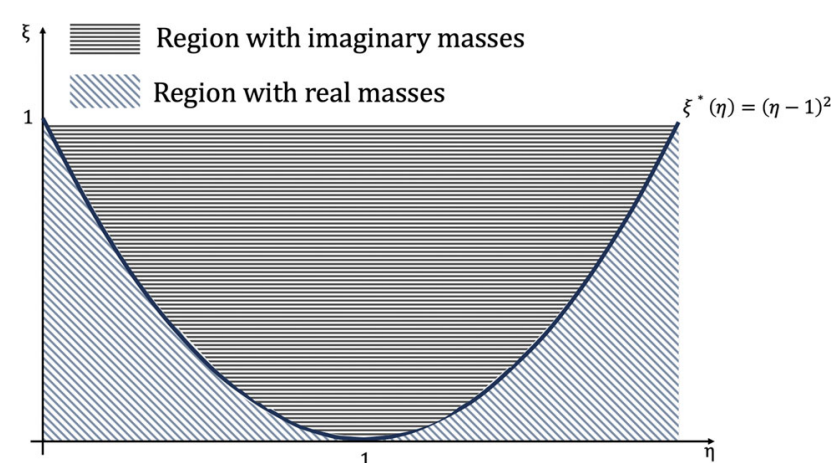

Fig. 1 Regions of the parameter space $(\eta, \xi)$ that admit models of STFOG with real masses $m_{ \pm}^{2} \geqslant 0:\{(\eta, \xi) \mid 0 \leqslant \eta<+\infty, 0 \leqslant \xi<$ $\left.\xi^{*}(\eta)\right\}$, where $\xi^{*}(\eta)=(\eta-1)^{2}$

where

$$
\begin{aligned}
g(\eta, \xi) & =\mathcal{A}(\eta, \xi)+\frac{\xi}{3 \sqrt{\eta^{4}+(\xi-1)^{2}-2 \eta^{2}(\xi+1)}} \\
& =\frac{1}{6}+\frac{1+\xi-\eta^{2}}{6 \sqrt{\eta^{4}+(\xi-1)^{2}-2 \eta^{2}(\xi+1)}}
\end{aligned}
$$

with $M$ the point-like mass. Since the field equations are linear, we can ever find the solution for a point-like source distribution, we get:

$$
\begin{aligned}
\Phi(\mathbf{x})= & -\frac{G}{f_{R}\left(0, \phi^{0}\right)} \sum_{i} \frac{m_{i}}{\left|\mathbf{x}-\mathbf{x}_{i}\right|}\left[1+g(\eta, \xi) e^{-m_{+}\left|\mathbf{x}-\mathbf{x}_{i}\right|}\right. \\
& \left.+[1 / 3-g(\eta, \xi)] e^{-m_{-}\left|\mathbf{x}-\mathbf{x}_{i}\right|}\right]
\end{aligned}
$$

This potential presents two contributions Yukawa-like characterized by two scale lengths defined by the masses $m_{ \pm}$.

\section{The virial theorem in fourth order gravity}

Let us consider a stellar system composed by $N$ stars with mass $m_{i}$. Considering the potential (12), the force between the $i$-th and $j$-th star is given:

$\mathcal{F}_{i j}=-m_{i} \nabla \Phi_{j}(\mathbf{x})$,

while the motion of $i$-th star is described by

$m_{i} \frac{d \mathbf{v}_{i}}{d t}=-\sum_{j=0}^{N} \frac{\tilde{G} m_{i} m_{j}\left(\mathbf{x}_{i}-\mathbf{x}_{j}\right)}{\left|\mathbf{x}_{i}-\mathbf{x}_{j}\right|^{3}}\left[1+\Lambda\left(\left|\mathbf{x}_{i}-\mathbf{x}_{j}\right|\right)\right]$,

where $\mu_{1}=m_{+}, \mu_{2}=m_{-}, \lambda_{1}=g(\eta, \xi), \lambda_{2}=$ $1 / 3-g(\eta, \xi), \tilde{G}=G / f_{R}\left(0, \phi^{(0)}\right)$ and $\Lambda\left(\left|\mathbf{x}_{i}-\mathbf{x}_{j}\right|\right)$ is the contribution of STFOG, given by

$\Lambda\left(\left|\mathbf{x}_{i}-\mathbf{x}_{j}\right|\right)=\sum_{k=1}^{2} \lambda_{k}\left(1+\mu_{k}\left|\mathbf{x}_{i}-\mathbf{x}_{j}\right|\right) e^{-\mu_{k}\left|\mathbf{x}_{i}-\mathbf{x}_{j}\right|}$. 
If we multiply the motion equation (14) by $\mathbf{x}_{i}$ and we sum, we get:

$$
\begin{aligned}
& \sum_{i=0}^{N} m_{i} \frac{d \mathbf{v}_{i}}{d t} \cdot \mathbf{x}_{i}=-\sum_{j=0}^{N} \frac{\tilde{G} m_{i} m_{j}\left(\mathbf{x}_{i}-\mathbf{x}_{j}\right) \cdot \mathbf{x}_{i}}{\left|\mathbf{x}_{i}-\mathbf{x}_{j}\right|^{3}} \\
& \quad \times\left[1+\Lambda\left(\left|\mathbf{x}_{i}-\mathbf{x}_{j}\right|\right)\right] .
\end{aligned}
$$

By substituting $i \rightleftarrows j$ we get:

$$
\begin{aligned}
& \sum_{i=0}^{N} m_{i} \frac{d \mathbf{v}_{i}}{d t} \cdot \mathbf{x}_{i} \\
& =-\frac{1}{2} \sum_{i, j=0}^{N} \frac{\tilde{G} m_{i} m_{j}}{\left|\mathbf{x}_{i}-\mathbf{x}_{j}\right|}\left[1+\Lambda\left(\left|\mathbf{x}_{i}-\mathbf{x}_{j}\right|\right)\right] .
\end{aligned}
$$

Consider the inertial momentum $I$ :

$I=\frac{1}{2} \sum_{i=0}^{N} m_{i} \mathbf{x}_{i} \cdot \mathbf{x}_{i}$

we note that

$\frac{d^{2} I}{d t^{2}}=\sum_{i=0}^{N} m_{i} \frac{d \mathbf{v}_{i}}{d t} \cdot \mathbf{x}_{i}+\sum_{i=0}^{N} m_{i}\left|\mathbf{v}_{i}\right|^{2}$,

where $\mathbf{v}_{i}=\frac{d \mathbf{x}_{i}}{d t}$. Using the relation (19), the Eq. (17) takes the form:

$$
\begin{aligned}
\frac{d^{2} I}{d t^{2}} & =\sum_{i=0}^{N} m_{i}\left|\mathbf{v}_{i}\right|^{2} \\
& -\frac{1}{2} \sum_{i, j=0 i \neq j}^{N} \frac{\tilde{G} m_{i} m_{j}}{\left|\mathbf{x}_{i}-\mathbf{x}_{j}\right|}\left[1+\Lambda\left(\left|\mathbf{x}_{i}-\mathbf{x}_{j}\right|\right)\right] .
\end{aligned}
$$

Let us recall that the kinetic energy $K$ and the Newtonian gravitational potential energy $U_{N}$ of stellar system are given by

$$
\begin{aligned}
& K=\sum_{i=0}^{N} m_{i}\left|\mathbf{v}_{i}\right|^{2}, \\
& U_{N}=-\frac{1}{2} \sum_{i, j=0 i \neq j}^{N} \frac{\tilde{G} m_{i} m_{j}}{\left|\mathbf{x}_{i}-\mathbf{x}_{j}\right|} .
\end{aligned}
$$

Hence,

$$
\frac{d^{2} I}{d t^{2}}=2 K+U_{N}+U_{S T F O G}
$$

where

$U_{S T F O G}=-\frac{1}{2} \sum_{i, j=0 i \neq j}^{N} \frac{\tilde{G} m_{i} m_{j}}{\left|\mathbf{x}_{i}-\mathbf{x}_{j}\right|} \Lambda\left(\left|\mathbf{x}_{i}-\mathbf{x}_{j}\right|\right)$,

and $\Lambda\left(\left|\mathbf{x}_{i}-\mathbf{x}_{j}\right|\right)$ given by Eq. (15). The expression (22) can be evaluated as temporal average on the interval $\tau$. The average quantity is obtained in the limit $\tau \rightarrow \infty$ and the averaging operation is given by $\langle\cdot\rangle=\lim _{\tau \rightarrow \infty} \frac{\int_{0}^{\tau} d t}{\tau}$. The average of Eq. (22) is then

$$
\frac{\frac{d I(\tau)}{d t}-\frac{d I(0)}{d t}}{\tau}=2\langle K\rangle+\left\langle U_{N}\right\rangle+\left\langle U_{S T F O G}\right\rangle .
$$

Since $\frac{d I}{d t}$ is a bounded function of time, hence the time average of $\left\langle\frac{d^{2} I}{d t^{2}}\right\rangle$ vanishes (for $\tau \rightarrow \infty$ we have $\frac{d I}{d t} \rightarrow 0$ ). This is based on the assumption that the velocities and the spatial coordinates of all stars remain finite for all time. Then, we obtain the expression of the generalized virial theorem in the tensor scalar fourth order gravity

$2\langle K\rangle+\left\langle U_{N}\right\rangle+\left\langle U_{S T F O G}\right\rangle=0$.

The deviation of the virial theorem in the Eq. (25) from the Newtonian result $\left(2\langle K\rangle+\left\langle U_{N}\right\rangle=0\right)$ is contained in $\left\langle U_{S T F O G}\right\rangle$, where $U_{S T F O G}$ is given by Eq. (23). More explicitly, we have

$$
\begin{aligned}
& U_{S T F O G}\left(\left|\mathbf{x}_{i}-\mathbf{x}_{j}\right|\right)=-\frac{\tilde{G}}{2} \sum_{k=1}^{2} \sum_{i, j=0 i \neq j}^{N} \\
& \times \frac{\lambda_{k} m_{i} m_{j}}{\left|\mathbf{x}_{i}-\mathbf{x}_{j}\right|}\left(1+\mu_{k}\left|\mathbf{x}_{i}-\mathbf{x}_{j}\right|\right) e^{-\mu_{k}\left|\mathbf{x}_{i}-\mathbf{x}_{j}\right|} \mid .
\end{aligned}
$$

Therefore, the fundamental difference between the Newtonian form of virial theorem [49] and the (25) is given by the presence of the term proportional to $e^{-\mu_{k}\left|\mathbf{x}_{i}-\mathbf{x}_{j}\right|}$ in Eq. (25). This presence is the outcome of no-validity of Gauss theorem in fourth order gravity. The gravitational energy $U_{S T ~ F O G}$ can be recast in the case of a continuous mass distribution as follows:

$$
\begin{gathered}
U_{S T F O G}\left(\left|\mathbf{x}_{i}-\mathbf{x}_{j}\right|\right)=-\frac{\tilde{G}}{2} \sum_{k=1}^{2} \lambda_{k} \int d^{3} \mathbf{x}^{\prime} d^{3} \mathbf{x}^{\prime \prime} \\
\times \frac{\rho\left(\mathbf{x}^{\prime}\right) \rho\left(\mathbf{x}^{\prime \prime}\right)}{\left|\mathbf{x}^{\prime}-\mathbf{x}^{\prime \prime}\right|}\left(1+\mu_{k}\left|\mathbf{x}^{\prime}-\mathbf{x}^{\prime \prime}\right|\right) e^{-\mu_{k}\left|\mathbf{x}^{\prime}-\mathbf{x}^{\prime \prime}\right|},
\end{gathered}
$$

where $\rho$ is the mass distribution of the stars in the stellar system.

\section{Effective dark matter}

The ordinary matter (baryonic matter) of which all stars and galaxies are made, only accounts for 5\% of the content of the universe. This matter absorb, reflect or emit light. However, most of the matter in the universe does not interact with the electromagnetic force. This means that it does not absorb, reflect or emit light, making it extremely hard to spot: dark matter. Then, the properties and the existence of this unknown form of matter can be analyzed only by means of its gravitational effect on the ordinary matter.

Currently, the mass-to-light ratio $M / L$ is used to estimate the baryonic mass for astrophysics systems. However, from 
the observations comes out that the dynamic mass of the system is usually larger than that estimated by mass-to-light ratio. For this reason, this experimental evidence is normally attributed to the possible existence of dark matter (nonbaryonic matter).

Let us indicate with $\mathcal{M}_{b m}$ and $\mathcal{M}_{d m}$ respectively the baryonic matter and the dark matter, and with $\varepsilon_{d m}=\mathcal{M}_{d m} / \mathcal{M}_{b m}$ their ratio. The virial mass (dynamic mass) $\mathcal{M}_{v}$ of the system can be written as $\mathcal{M}_{v}=\mathcal{M}_{b m}+\mathcal{M}_{d m}=\mathcal{M}_{b m}\left(1+\varepsilon_{d m}\right)$. In astronomy, assuming the validity of the virial theorem, the virial mass is defined as the mass of a gravitationally bound astrophysical system. For sufficiently isolated selfgravitating astronomical systems in virial equilibrium, the virial theorem employed in astronomy, has the following expression $[50,51]$ :

$2\langle K\rangle+\left(1+\varepsilon_{d m}\right)\left\langle U_{N}\right\rangle=0$.

In weak-field approximation, the STFOG presents some additional terms in the virial theorem (25) that they could simulate the amount of dark matter. Indeed, comparing Eq. (25) with Eq. (28) the STFOG could account for this mass excess provided:

$\left\langle U_{S T F O G}\right\rangle=\varepsilon_{d m}\left\langle U_{N}\right\rangle$,

where $U_{S T F O G}$ and $U_{N}$ are given respectively in Eqs. (26) and (21). Therefore, we are assuming that the additional contributions of STFOG (26) contribute to the dynamics of the astronomical systems, in virial equilibrium, as effective dark matter. Explicitly, Eq. (29) takes the form

$$
\begin{aligned}
& \left\langle\sum_{k=1}^{2} \lambda_{k} \int d^{3} \mathbf{x}^{\prime} d^{3} \mathbf{x}^{\prime \prime} \frac{\rho\left(\mathbf{x}^{\prime}\right) \rho\left(\mathbf{x}^{\prime \prime}\right)}{\left|\mathbf{x}^{\prime}-\mathbf{x}^{\prime \prime}\right|}\left(1+\mu_{k}\left|\mathbf{x}^{\prime}-\mathbf{x}^{\prime \prime}\right|\right) e^{-\mu_{k}\left|\mathbf{x}^{\prime}-\mathbf{x}^{\prime \prime}\right|}\right\rangle \\
& \quad=\varepsilon_{d m}\left\langle\int d^{3} \mathbf{x}^{\prime} d^{3} \mathbf{x}^{\prime \prime} \frac{\rho\left(\mathbf{x}^{\prime}\right) \rho\left(\mathbf{x}^{\prime \prime}\right)}{\left|\mathbf{x}^{\prime}-\mathbf{x}^{\prime \prime}\right|}\right\rangle
\end{aligned}
$$

Introducing the following function $F\left(\mu_{k}\right)$ (see Appendix A)

$$
\begin{aligned}
F\left(\mu_{k}\right)= & \int d^{3} \mathbf{x}^{\prime} d^{3} \mathbf{x}^{\prime \prime} \frac{\rho\left(\mathbf{x}^{\prime}\right) \rho\left(\mathbf{x}^{\prime \prime}\right)}{\left|\mathbf{x}^{\prime}-\mathbf{x}^{\prime \prime}\right|} \\
& \times\left(1+\mu_{k}\left|\mathbf{x}^{\prime}-\mathbf{x}^{\prime \prime}\right|\right) e^{-\mu_{k}\left|\mathbf{x}^{\prime}-\mathbf{x}^{\prime \prime}\right|},
\end{aligned}
$$

Eq. (30) can be rewritten as

$$
\left\langle\sum_{k=1}^{2} \lambda_{k} F\left(\mu_{k}\right)\right\rangle=\varepsilon_{d m}\langle F(0)\rangle .
$$

Equation (32) is an algebraic relation between the free parameters contained in a STFOG, $\mu_{1}$ and $\mu_{2}$, and the ratio between dark matter and baryon matter, i.e. $\varepsilon_{d m}$. In general, the relation (32) represents a condition that the STFOG must satisfy in order to describe the presence of dark matter in the isolated self-gravitating astronomical systems in virial equilibrium. The inconsistency of the relation (32) can be considered as a criterion for excluding models of STFOG as hypothetical mechanisms to generate and describe the presence of dark matter in the astronomical systems above considered.

\section{Observational constraints}

There are several astronomical observations, for a sufficiently isolated galaxy in virial equilibrium, with high percentage of dark matter, i.e. $\varepsilon_{d m}>1$. As an example we have the Andromeda Galaxy with $\varepsilon_{d m}=12.7$ [52-54]; the Triangulum Galaxy with $\varepsilon_{d m}=5$ [55]; NGC1047 Galaxy with $\varepsilon_{d m}=31$ [56].

Now let's analyse the consistency of the relation (32) for some models of STFOG studied in literature (Table 1):

- Case A: the relation (32) for a sample $f(R)$-theory (see Fig. 2) becomes:

$$
\left\langle\frac{1}{3} F\left(m_{R}\right)\right\rangle=\varepsilon_{d m}\langle F(0)\rangle .
$$

In this case, using the properties of the function $F(x)$ (see Appendix A) and the condition $\varepsilon_{d m}>1$, the algebraic relation (33) does not admit any solution. Indeed, one has:

$\frac{\left\langle F\left(m_{R}\right)\right\rangle}{\langle F(0)\rangle}<1$ and $3 \varepsilon_{d m}>1$.

Therefore, the $f(R)$ models could explain the presence of dark matter only in galaxies with low percentage of dark matter. Recent observations have shown that some galactic globular clusters [57] contain a relatively low percentage of effective dark matter, $\varepsilon_{d m}=0.4$. However, for these systems the hypothesis of isolated selfgravitating astronomical systems in virial equilibrium may not be verified. In general, the influence of a much larger neighboring galaxy on the dynamics of the globular clusters cannot be ignored. The analysis of this system goes beyond the scope of this paper.

- Case B: the relation (32) for these models (see Fig. 3) takes the form:

$\left\langle\left[\frac{1}{3}-g(0, \xi)\right] F\left(m_{-}\right)\right\rangle=\varepsilon_{d m}\langle F(0)\rangle$.

Also in this case we never have a solution, because the left-hand side of the equation (34) is always negative $\forall \xi(0<\xi<1)$, while the right-hand side is positive. 
Table 1 Some models of STFOG

\begin{tabular}{|c|c|c|}
\hline Case & STFOG & Parameters \\
\hline A & $f(R)$ & $\begin{array}{l}m_{R}^{2}=-\frac{f_{R}\left(0,0, \phi^{(0)}\right)}{3 f_{R R}(0)}, \quad m_{\phi}^{2}=0 \\
\eta=0, \quad \xi=0 \\
m_{+}=m_{R}, \quad m_{-}=0 \\
\lambda_{1}=\frac{1}{3}, \quad \lambda_{2}=0\end{array}$ \\
\hline B & $R+\alpha \phi R+f(\phi)+\omega(\phi) \phi_{; \alpha} \phi^{; \alpha}$ & $\begin{array}{l}m_{R}^{2} \rightarrow+\infty, m_{\phi}^{2}=-\frac{f_{\phi \phi}\left(0, \phi^{(0)}\right)}{2 \omega\left(\phi^{(0)}\right)} \\
\eta \rightarrow 0, \quad \xi=\frac{3 \alpha^{2}}{2 \omega\left(\phi^{(0)}\right)} \\
m_{+} \rightarrow+\infty, \quad m_{-} \rightarrow \frac{m_{\phi}}{\sqrt{1-\xi}} \\
\lambda_{1}=g(0, \xi)>0, \quad \lambda_{2}=1 / 3-g(0, \xi)<0 \\
m_{R}^{2}=-\frac{f_{R}\left(0,0, \phi^{(0)}\right)}{3 f_{R R}(0)}, \quad m_{\phi}^{2}=-\frac{f_{\phi \phi}\left(0, \phi^{(0)}\right)}{2 \omega\left(\phi^{(0)}\right)}\end{array}$ \\
\hline $\mathrm{C}$ & $f(R, \phi)+\omega(\phi) \phi_{; \alpha} \phi^{; \alpha}$ & $\begin{array}{l}\eta=\frac{m_{\phi}}{m_{R}}, \quad \xi=\frac{3 f_{R \phi}\left(0, \phi^{(0)}\right)^{2}}{2 \omega\left(\phi^{(0)}\right)} \\
m_{+}=\omega_{+} m_{R}, \quad m_{-}=\omega_{-} m_{R}, \\
\eta<1 \Rightarrow \lambda_{1}=g(\eta, \xi)>0, \quad \lambda_{2}=1 / 3-g(\eta, \xi)<0 \\
\eta>1 \Rightarrow \lambda_{1}=g(\eta, \xi)<0, \quad \lambda_{2}=1 / 3-g(\eta, \xi)>0\end{array}$ \\
\hline
\end{tabular}

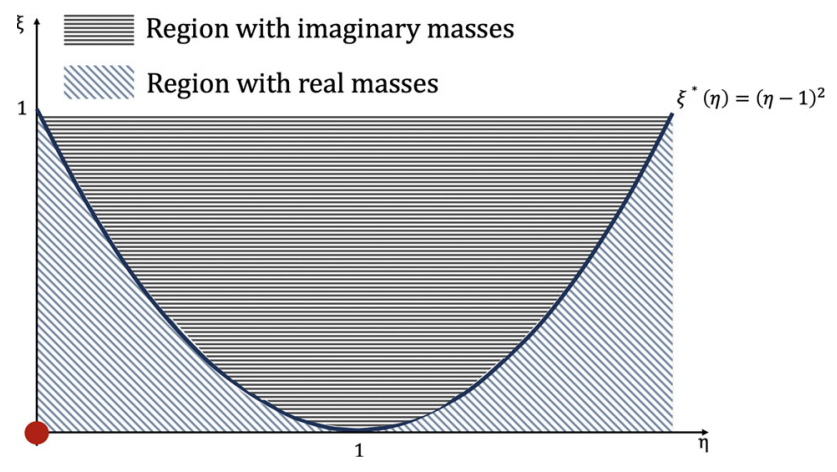

Fig. 2 In the space defined by parameters $(\eta, \xi)$ some classes of models (1) could be represented with a representative point in the $\eta \xi$-plan. On the abscissa axis $(\xi=0)$ are representable STFOG models with a minimally coupled $\left(\xi=0 \Rightarrow f_{R \phi}=0\right.$ ), while on the ordinate axis $(\eta=0)$ are represented STFOG models with either a massless scalar field ( $\eta=0 \Rightarrow m_{\phi}=0$ ) or models with a massive scalar field nonminimally coupled in General Relativity $\left(\eta=0 \Rightarrow m_{R} \rightarrow \infty\right)$. In the origin of the axes, $(\eta, \xi) \equiv(0,0)$, are representable all $f(R)$-models

Therefore, also these models are not compatible with the presence of dark matter in the galaxies.

- Case C: the relation (32) in this case assumes the following more general expression:

$$
\left\langle g(\eta, \xi) F\left(m_{+}\right)+\left[\frac{1}{3}-g(\eta, \xi)\right] F\left(m_{-}\right)\right\rangle=\varepsilon_{d m}\langle F(0)\rangle .
$$

Now we analyse the possible solutions of the Eq. (35) distinguishing two cases: $\eta>1$ and $\eta<1$. In the first case,

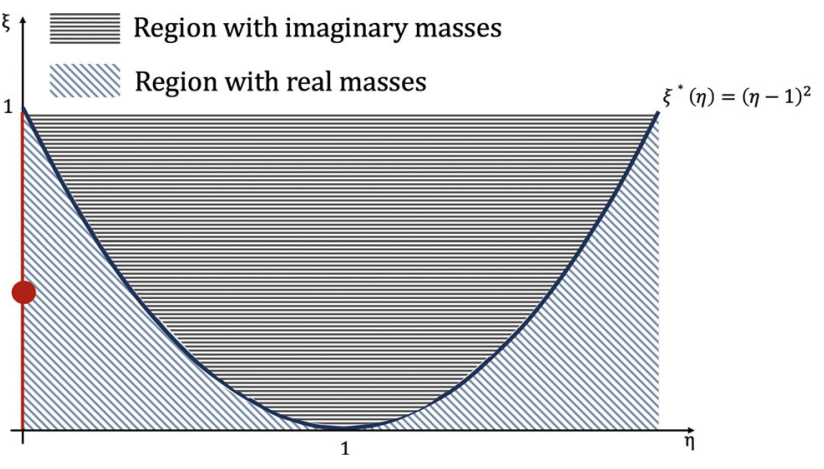

Fig. 3 Models with the following structure $R+\alpha \phi R+f(\phi)+$ $\omega(\phi) \phi_{; \alpha} \phi^{; \alpha}$ can be represented in $\eta \xi$-plan as a point of the segment (in red): $(\eta, \xi) \equiv(0, \xi)$, with $0<\xi<1$

i.e. $\eta>1$, the models considered can be represented in the $\eta \xi$-plan as shown in the Fig. 4a. After some algebra, using the properties of the function $F(x)$ (see Appendix A), we obtain that the Eq. (35) admits a solution only if:

$g(\eta, \xi) \leqslant \frac{1}{3}-\varepsilon_{d m}$.

For a generic value of $\xi \neq \xi^{*}(\eta)$ (see Fig. 4a), as shown in Fig. 4b we have:

$-3 \leqslant g(\eta, \xi) \leqslant 0$

while using the observed values [52-56] for the ratio $\varepsilon_{d m}$, we get:

$-30.7 \leqslant \frac{1}{3}-\varepsilon_{d m} \leqslant-4.7$.

Therefore, the Eq. (35) does not admit solution. 


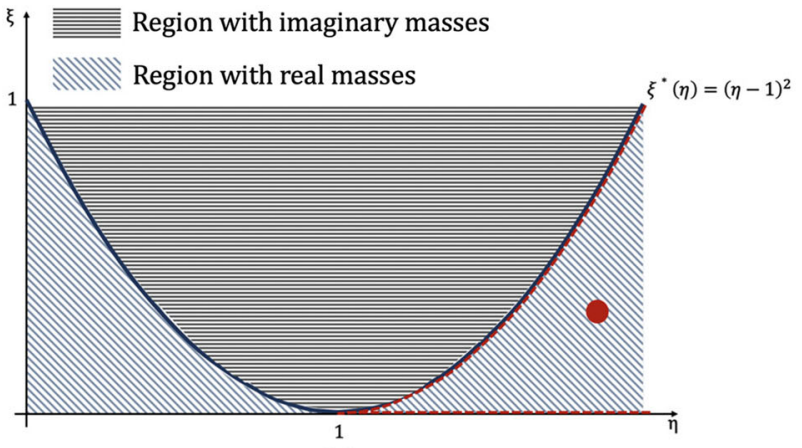

(a)

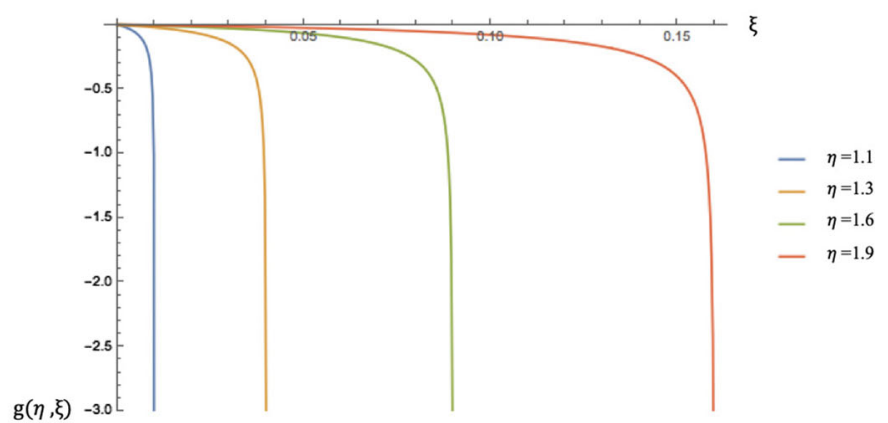

(b)

Fig. 4 a Models with the structure $f(R, \phi)+\omega(\phi) \phi_{; \alpha} \phi^{; \alpha}$ can be represented in $\eta \xi$-plan as the generic point (red): $\{(\eta, \xi) \mid \eta>1,0<\xi<$ $\left.\xi^{*}(\eta)\right\}$; b graph of the function $g(\eta, \xi)$ with $\eta>1$

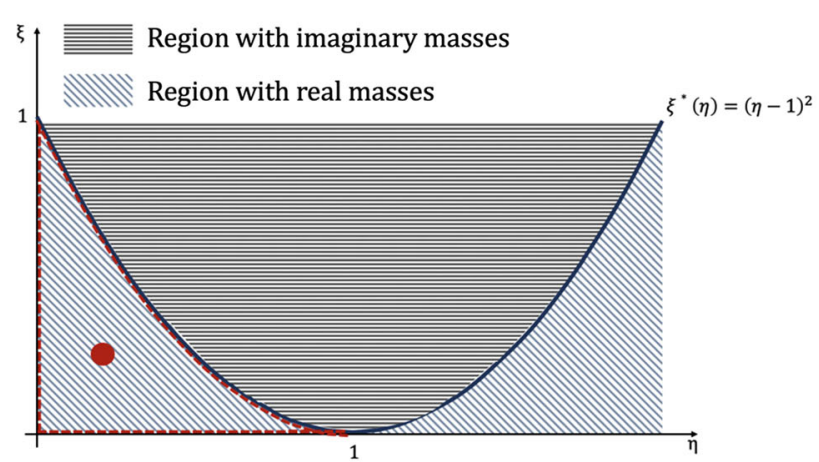

(a)

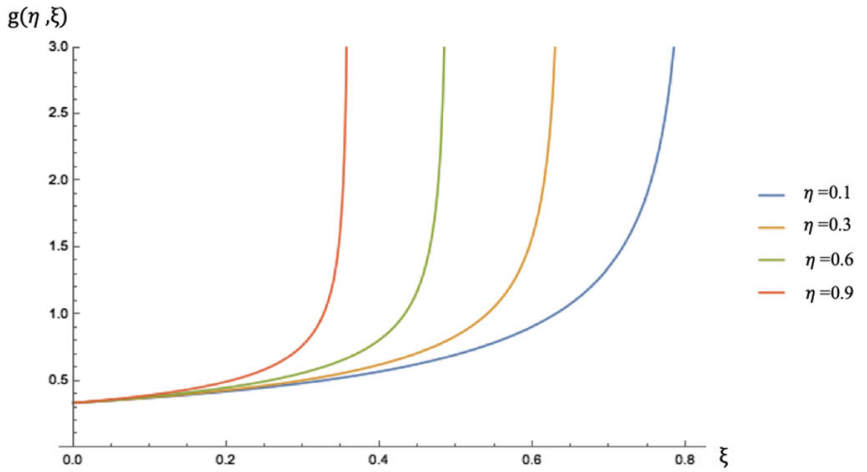

(b)

Fig. 5 a Models with the structure $f(R, \phi)+\omega(\phi) \phi_{; \alpha} \phi^{; \alpha}$ can be represented in $\eta \xi$-plan as the generic point (red): $\{(\eta, \xi) \mid \eta<1,0<\xi<$ $\left.\xi^{*}(\eta)\right\}$; b graph of the function $g(\eta, \xi)$ with $\eta<1$

In the second case, i.e. for $\eta<1$, the models considered can be represented as shown in the Fig. 5a. As in the previous case, after straightforward computations, it is possible to prove that there is never a solution when is satisfied the following condition:

$\frac{\varepsilon_{d m}}{g(\eta, \xi)}>1$

On the other hand, a solution is admitted for

$\frac{\varepsilon_{d m}}{g(\eta, \xi)}<1$.

Moreover, for a generic value of $\xi \neq \xi^{*}(\eta)$ (see Fig. 5a), as shown in Fig. 5b we have:

$\frac{1}{3} \leqslant g(\eta, \xi) \leqslant 3$,

while using the observed values [52-56] for the ratio $\varepsilon_{d m}$, we get:

$5 \leqslant \varepsilon_{d m} \leqslant 31$.
Therefore, the Eq. (35) does not admit solution also in this case.

\section{Conclusions}

We have analysed the virial theorem for sufficiently isolated galaxy in virial equilibrium, at the present time. We have considered the Newtonian limit in the context of STFOG. In this framework, we have shown that for a point-like source distribution, the newtonian potential presents two contributions Yukawa-like characterized by two scale lengths defined by the masses $m_{ \pm}$. These masses depend on the gravity model considered through the parameters $\eta$ and $\xi$ and we have shown the regions of the parameter space $(\eta, \xi)$ in which $m_{ \pm}$assume real values (cf. Fig. 1).

We have obtained the expression of the generalized virial theorem in the STFOG, (Eq. (25)), in which an extra contribution, namely $\left\langle U_{S T F O G}\right\rangle$, presents a term proportional to $e^{-\mu_{k}\left|\mathbf{x}_{i}-\mathbf{x}_{j}\right|}$, due to the no-validity of Gauss theorem in 
STFOG. We have interpreted $\left\langle U_{S T F O G}\right\rangle$ as a term that could simulate the amount of dark matter: $\left\langle U_{S T F O G}\right\rangle=\varepsilon_{d m}\left\langle U_{N}\right\rangle$. In other words, this additional extra contribution in STFOG could be seen as effective dark matter.

We derived an algebraic relation between the free parameters contained in a STFOG, and the ratio between dark matter and baryon matter (Eq. (32)), and we have shown that for some models of STFOG such relation does not admit solutions for isolated galaxies in virial equilibrium with a large fraction of dark matter, i.e. $\varepsilon_{d m}>1$. For these reasons, STFOG is not compatible with the amount of dark matter contained in these astronomical systems necessary to explain the flatness of the galaxies rotation curves. Therefore, some other mechanism must be considered in order interpret correctly the experimental data.

Data Availability Statement This manuscript has no associated data or the data will not be deposited. [Authors' comment: The experimental data used in this work can be taken from experimental papers Ref. [[5257].]

Open Access This article is licensed under a Creative Commons Attribution 4.0 International License, which permits use, sharing, adaptation, distribution and reproduction in any medium or format, as long as you give appropriate credit to the original author(s) and the source, provide a link to the Creative Commons licence, and indicate if changes were made. The images or other third party material in this article are included in the article's Creative Commons licence, unless indicated otherwise in a credit line to the material. If material is not included in the article's Creative Commons licence and your intended use is not permitted by statutory regulation or exceeds the permitted use, you will need to obtain permission directly from the copyright holder. To view a copy of this licence, visit http://creativecomm ons.org/licenses/by/4.0/.

Funded by SCOAP ${ }^{3}$.

\section{Appendix A: Properties of the function $F$}

In Sect. 4, we have introduced the function $F(\mu)$, Eq. (31), defined as:

$$
\begin{aligned}
& F(\mu)=\int d^{3} \mathbf{x}^{\prime} d^{3} \mathbf{x}^{\prime \prime} \\
& \times \frac{\rho\left(\mathbf{x}^{\prime}\right) \rho\left(\mathbf{x}^{\prime \prime}\right)}{\left|\mathbf{x}^{\prime}-\mathbf{x}^{\prime \prime}\right|}\left(1+\mu\left|\mathbf{x}^{\prime}-\mathbf{x}^{\prime \prime}\right|\right) e^{-\mu\left|\mathbf{x}^{\prime}-\mathbf{x}^{\prime \prime}\right|} .
\end{aligned}
$$

It is easy to notice that $F(\mu)$ is a continuous, limited and positive function of the variable $\mu$, defined $\forall \mu \in[0,+\infty[$. In its domain $F(\mu)$ is a decreasing monotonic function with limit 0 when $\mu \rightarrow \infty$.

Let's consider the Maclaurin series of the function $F(\mu)$ :

$F(\mu)=F(0)+\sum_{n=1}^{\infty} \frac{F^{(n)}(0)}{n !} \mu^{n}$, where $\left.F^{(n)}(0) \equiv \frac{d^{n} F(\mu)}{d \mu^{n}}\right|_{\mu=0}$. The expression of $F(0)$ takes the form:

$F(0)=\int d^{3} \mathbf{x}^{\prime} d^{3} \mathbf{x}^{\prime \prime} \frac{\rho\left(\mathbf{x}^{\prime}\right) \rho\left(\mathbf{x}^{\prime \prime}\right)}{\left|\mathbf{x}^{\prime}-\mathbf{x}^{\prime \prime}\right|}$,

instead, the generic term of $n$th derivative is:

$$
\begin{aligned}
& \frac{d^{n} F(\mu)}{d \mu^{n}}=(-1)^{(n-1)}(n-1) \\
& \quad \times \int d^{3} \mathbf{x}^{\prime} d^{3} \mathbf{x}^{\prime \prime} \rho\left(\mathbf{x}^{\prime}\right) \rho\left(\mathbf{x}^{\prime \prime}\right)\left|\mathbf{x}^{\prime}-\mathbf{x}^{\prime \prime}\right|^{(n-1)} e^{-\mu\left|\mathbf{x}^{\prime}-\mathbf{x}^{\prime \prime}\right|} \\
& +(-1)^{n} \mu \int d^{3} \mathbf{x}^{\prime} d^{3} \mathbf{x}^{\prime \prime} \rho\left(\mathbf{x}^{\prime}\right) \rho\left(\mathbf{x}^{\prime \prime}\right)\left|\mathbf{x}^{\prime}-\mathbf{x}^{\prime \prime}\right|^{n} e^{-\mu\left|\mathbf{x}^{\prime}-\mathbf{x}^{\prime \prime}\right|} .
\end{aligned}
$$

In particular, the first and the second derivative are:

$$
\begin{aligned}
\frac{d F(\mu)}{d \mu}= & -\mu \int d^{3} \mathbf{x}^{\prime} d^{3} \mathbf{x}^{\prime \prime} \rho\left(\mathbf{x}^{\prime}\right) \rho\left(\mathbf{x}^{\prime \prime}\right)\left|\mathbf{x}^{\prime}-\mathbf{x}^{\prime \prime}\right| e^{-\mu\left|\mathbf{x}^{\prime}-\mathbf{x}^{\prime \prime}\right|}, \\
\frac{d^{2} F(\mu)}{d \mu^{2}}= & -\int d^{3} \mathbf{x}^{\prime} d^{3} \mathbf{x}^{\prime \prime} \rho\left(\mathbf{x}^{\prime}\right) \rho\left(\mathbf{x}^{\prime \prime}\right)\left|\mathbf{x}^{\prime}-\mathbf{x}^{\prime \prime}\right| e^{-\mu\left|\mathbf{x}^{\prime}-\mathbf{x}^{\prime \prime}\right|} \\
& +\mu \int d^{3} \mathbf{x}^{\prime} d^{3} \mathbf{x}^{\prime \prime} \rho\left(\mathbf{x}^{\prime}\right) \rho\left(\mathbf{x}^{\prime \prime}\right)\left|\mathbf{x}^{\prime}-\mathbf{x}^{\prime \prime}\right|^{2} e^{-\mu\left|\mathbf{x}^{\prime}-\mathbf{x}^{\prime \prime}\right|} .
\end{aligned}
$$

From the study of the sign of the first derivatives (A5), we note that the function $F(\mu)$ is strictly decreasing:

$\frac{d F(\mu)}{d \mu} \leqslant 0$ when $\mu \geqslant 0$.

Then, the function $F(\mu)$ has a global maximum in $\mu=0$ :

$F(\mu) \leq F(0), \quad \forall \mu$.

While from the study of the sign of the second derivative (A6), the function $F(\mu)$ has an inflection point $\mu_{f}$, (with $\left.0<\mu_{f}<1\right)$ :

$\frac{d^{2} F(\mu)}{d \mu^{2}} \geq 0$ when $\mu \geq \mu_{f}$

Note that $\mu_{f}$ is defined by the following constitutive equation:

$$
\begin{aligned}
& \int d^{3} \mathbf{x}^{\prime} d^{3} \mathbf{x}^{\prime \prime} \rho\left(\mathbf{x}^{\prime}\right) \rho\left(\mathbf{x}^{\prime \prime}\right)\left|\mathbf{x}^{\prime}-\mathbf{x}^{\prime \prime}\right| e^{-\mu_{f}\left|\mathbf{x}^{\prime}-\mathbf{x}^{\prime \prime}\right|} \\
& =\mu_{f} \int d^{3} \mathbf{x}^{\prime} d^{3} \mathbf{x}^{\prime \prime} \rho\left(\mathbf{x}^{\prime}\right) \rho\left(\mathbf{x}^{\prime \prime}\right)\left|\mathbf{x}^{\prime}-\mathbf{x}^{\prime \prime}\right|^{2} e^{-\mu_{f}\left|\mathbf{x}^{\prime}-\mathbf{x}^{\prime \prime}\right|}
\end{aligned}
$$

From the Eq. (A4) we get:

$$
\begin{aligned}
& F^{(n)}(0)=(-1)^{(n-1)}(n-1) \\
& \quad \times \int d^{3} \mathbf{x}^{\prime} d^{3} \mathbf{x}^{\prime \prime} \rho\left(\mathbf{x}^{\prime}\right) \rho\left(\mathbf{x}^{\prime \prime}\right)\left|\mathbf{x}^{\prime}-\mathbf{x}^{\prime \prime}\right|^{(n-1)}, \quad \forall n \geq 2 .
\end{aligned}
$$


We introduce the generator $\Theta(\mu)$ defined as:

$\Theta(\mu)=\int d^{3} \mathbf{x}^{\prime} d^{3} \mathbf{x}^{\prime \prime} \rho\left(\mathbf{x}^{\prime}\right) \rho\left(\mathbf{x}^{\prime \prime}\right) e^{-\mu\left|\mathbf{x}^{\prime}-\mathbf{x}^{\prime \prime}\right|}$

In this way, we can redefine the relation (A9) as follows:

$F^{n}(0)=\left.(n-1) \frac{d^{n-1} \Theta(\mu)}{d \mu^{n-1}}\right|_{\mu=0}, \quad \forall n \geq 1$.

Therefore, the Maclaurin series of function $F(\mu)$ takes the following expression:

$F(\mu)=F(0)+\left.\sum_{n=2}^{\infty} \frac{(n-1)}{n !} \frac{d^{n-1} \Theta(\mu)}{d \mu^{n-1}}\right|_{\mu=0} \mu^{n}$.

\section{References}

1. A.G. Riess et al., Astron. J. 116, 1009-1038 (1998)

2. S. Perlmutter et al., Astrophys. J. 517, 565-586 (1999)

3. S. Cole et al., Mon. Not. R. Astron. Soc. 362, 505-534 (2005)

4. D.N. Spergel et al., Astrophys. J. Suppl. Ser. 170, 377-408 (2007)

5. S.M. Carroll, W.H. Press, E.L. Turner, Annu. Rev. Astron. Astrophys. 30, 499-542 (1992)

6. V. Sahni, A. Starobinski, Int. J. Mod. Phys. D 9, 373-443 (2000)

7. J.F. Navarro, C.S. Frenk, S.D.M. White, Astrophys. J. 462, 563575 (1996)

8. S. Nojiri, S.D. Odintsov, Phys. Rep. 505, 59-104 (2011)

9. G. Olmo, Int. J. Mod. Phys. D 20, 413-462 (2011)

10. A. Stabile, G. Scelza, Phys. Rev. D 84(11), 124023 (2012)

11. I. Quandt, H.J. Schmidt, Astron. Nachr. 312, 97 (1991)

12. P. Teyssandier, Class. Quantum Gravity 6, 219 (1989)

13. C.M. Will, Theory and Experiment in Gravitational Physics, 2nd edn. (Cambridge University Press, Cambridge, 1993)

14. S. Capozziello, M. De Laurentis, Extended theories of gravity. Phys. Rep. 509, 167 (2011)

15. G.M. Tino, L. Cacciapuoti, S. Capozziello, G. Lambiase, F. Sorrentino, Prog. Part. Nucl. Phys. 112, 103772 (2020)

16. A. Stabile, Phys. Rev. D 82(12), 064021 (2010)

17. S. Capozziello, A. Stabile, Classical and Quantum Gravity: Theory, Analysis and Applications, chapter 2, (Nova Science Publishers, Inc, New York, 2010). ISBN:978-1-61122-957-8

18. S. Capozziello, A. Stabile, A. Troisi, Phys. Rev. D 76, 104019 (2007)

19. A. Stabile, S. Capozziello, Phys. Rev. D 87, 064002 (2013)

20. G. Lambiase, M. Sakellariadou, A. Stabile, JCAP 1312, 020 (2013)

21. S. Capozziello, G. Lambiase, M. Sakellariadou, A. Stabile, Phys. Rev. D 91, 044012 (2015)

22. S. Capozziello, G. Lambiase, M. Sakellariadou, A. Stabile, An. Stabile, Phys. Rev. D 91, 044012 (15) (2015)

23. L. Buoninfante, G. Lambiase, A. Stabile, Eur. Phys. J. C 80, 122 (2020)
24. G. Lambiase, M. Sakellariadou, A. Stabile, An Stabile, JCAP 1507(07), 003 (2015)

25. G. Lambiase, M. Sakellariadou, An. Stabile, JCAP 3, 14(2021)

26. A. Stabile, An Stabile, Phys. Rev. D 85, 044014 (2012)

27. S. Capozziello, A. Stabile, Astrophys. Space Sci. 358, 27 (2015)

28. C.G. Boehmer, T. Harko, F.S.N. Lobo, Astropart. Phys. 29, 386$392(2008)$

29. C.G. Boehmer, T. Harko, F.S.N. Lobo, J. Cosmol. Astropart. Phys. 0803, 024 (2008)

30. A. Stabile, G. Scelza, Astrophys. Space Sci. 357, 44 (2015)

31. A. Stabile, An. Stabile, S. Capozziello, Phys. Rev. D 88, 124011(9) (2013)

32. H. Weyl Raum zeit Materie: Vorlesungen uuber allgemeine Relativitatstheorie,(Springer, Berlin, 1921)

33. A.S. Eddington, The Mathematical Theory of Relativity, (Cambridge University Press, London, 1924)

34. C. Lanczos, Zeitschrift fur Physik A Hadrons and Nuclei 73, 147168 (1932)

35. W. Pauli, Phys. Zeit. 20, 457-467 (1919)

36. R. Bach, Zur Weylschen Relativitatstheorie und der Weylschen Erweiterung des Krummungstensorbegriffs. Mathematische Zeitschrift 9, 110-135 (1921)

37. H.A. Buchdahl, Il Nuovo Cimento 23, 141-157 (1962)

38. G.V. Bicknell, J. Phys. A Math. Nucl. Gen. 7, 1061 (1974)

39. N. Radicella, G. Lambiase, L. Parisi, G. Vilasi, JCAP 1412, 014 (2014)

40. S. Capozziello, G. Lambiase, Int. J. Mod. Phys. D 12, 843 (2003)

41. S.Calchi Novati, S. Capozziello, G. Lambiase, Gravit. Cosmol. 6, $173(2000)$

42. S. Capozziello, G. Lambiase, H.J. Schmidt, Ann. Phys. 9, 39 (2000)

43. S. Capozziello, G. Lambiase, G. Papini, G. Scarpetta, Phys. Lett. A 254, 11 (1999)

44. G. Lambiase, S. Mohanty, A. Stabile, Eur. Phys. J. C 78, 350 (2018)

45. G. Lambiase, A. Stabile, An Stabile, Phys. Rev. D 95, 084019 (2017)

46. M. Blasone, G. Lambiase, L. Petruzziello, A. Stabile, Eur. Phys. J. C 78(11), 976 (2018)

47. L. Buoninfante, G. Lambiase, L. Petruzziello, A. Stabile, Eur. Phys. J. C 79(1), 41 (2019)

48. K.S. Stelle, Phys. Rev. D 16, 953-969 (1977)

49. L. Binney, S. Tremaine, Galactic Dynamics (Princeton University Press, Princeton, 1994)

50. F. Zwicky, Helv. Phys. Acta 6, 110-127 (1933)

51. F. Zwicky, Astrophys. J. 86, 217-246 (1937)

52. P. Barmby et al., Astrophys. J. 650, L45-L49 (2006)

53. P. Barmby et al., Astrophys. J. 655, L61-L61 (2007)

54. A. Tamm, E. Tempel, P. Tenjes, O. Tihhonova, T. Tuvikene, Astron. Astrophys. 546, A4 (2012)

55. E. Corbelli, Mon. Not. R. Astron. Soc. 342, 199-207 (2003)

56. V. Pota, A.J. Romanowsky, J.P. Brodie, J. Penarrubia, D.A. Forbes, N.R. Napolitano, C. Foster, M.G. Walker, J. Strader, J.C. Roediger, Mon. Not. R. Astron. Soc. 450, 3345-3358 (2015)

57. A. Sollima, M. Bellazzini, J.-W. Lee, Astrophys. J. 755, 156 (2012) 